\title{
UAV-mounted GPR for NDT applications
}

\author{
Maria Garcia-Fernandez ${ }^{\#}$, Yuri Alvarez-Lopez ${ }^{\#}$, Borja Gonzalez-Valdes*, Ana Arboleya-Arboleya ${ }^{\dagger}$, \\ Yolanda Rodriguez-Vaqueiro*, Fernando Las Heras", Antonio Pino* \\ \#Area of Signal Theory and Communications, University of Oviedo, Gijón (Spain) \\ * Department of Signal Theory and Communications, University of Vigo, Vigo (Spain) \\ ${ }^{\dagger}$ Area of Signal Theory and Communications, University Rey Juan Carlos, Madrid (Spain) \\ mariagarcia@tsc.uniovi.es
}

\begin{abstract}
This contribution introduces a novel airborne system for subsurface sensing and imaging applications. The system consists of a Ground Penetrating Radar (GPR) mounted on an Unmanned Aerial Vehicle (UAV). Since the system does not need to be in contact with the soil, it is particularly useful for some Non-Destructive Testing (NDT) applications such as landmine detection or archeological surveys. An overview of the system and some of the first flight tests are shown in this contribution. The results of these flight tests prove the feasibility of the system to detect both metallic and dielectric targets. Furthermore, since the system includes a high-accuracy positioning system, measurements could be coherently combined to improve the radar image resolution.
\end{abstract}

Keywords - Ground Penetrating Radar (GPR), Unmanned Aerial Vehicle (UAV), Non-Destructive Testing (NDT), subsurface sensing.

\section{INTRODUCTION}

Detection of hidden objects in mediums opaque to the visible light using non-invasive techniques has raised an increasing interest in the last decades. These techniques have been successfully used for Non-Destructive Testing (NDT) applications in a wide range of fields such as civil engineering (structural damages and road inspection), security and defense (tunnel location and explosives detection), archeology, or search and rescue operations. They allow to detect, locate and obtain images from hidden objects avoiding the interaction with the medium and the objects.

Non-invasive techniques can be classified according to the physical phenomenon behind them [1]: electromagnetic induction, thermal sensors, nuclear quadrupole resonance and Ground Penetrating Radar (GPR). GPR has been considered a good candidate for subsurface sensing due to its capability to generate images from the underground and to detect both metallic and dielectric targets [2]. On the other hand, the main difficulties for detecting hidden targets using GPR are due to the ground roughness, the soil inhomogeneities and the possible low dielectric contrast between the underground and the targets.

GPR systems can be classified, according to the distance between the antennas and the soil, in ground-coupled or air-launched systems. The former are less affected by the strong reflections produced at the air-soil interface (due to impedance mismatching) and they can penetrate better into the soil. However, the time needed for a whole inspection is usually higher and they must be in contact with the soil.
This may damage buried objects in archeological surveys and it might be dangerous for some applications such as the detection of Improvised Explosive Devices (IEDs) and landmines. Therefore, for the detection of these kind of targets, air-launched systems are usually preferred in order to avoid accidental detonations. Air-launched systems can be further classified in Forward-Looking GPR (FLGPR) and Down-Looking GPR (DLGPR). In the former, the antennas look ahead of the vehicle, reducing reflections from the ground surface at the expense of a lower resolution [3]. In the latter, the antennas are perpendicular to the soil, achieving high resolution but suffering from strong specular reflections [4].

In DLGPR systems, the main challenge is to illuminate the soil with normal incidence and, at the same time, keep the safety distance. One approach is to use small lightweight terrestrial robots, with a small detonation risk and a low scanning speed. In order to minimize the risk and increase the speed, our approach, shown in Fig. 1, is based on mounting a GPR on an Unmanned Aerial Vehicle (UAV) [5]. Thus, the proposed system overcomes the main limitations of similar systems for the same purpose. It allows the safe and fast inspection of difficult-to-access areas, avoiding the contact with the soil. It also makes possible the detection of both metallic and dielectric targets (due to the use of a radar instead of a metal detector, such as in [6]). Furthermore, since the UAV includes a high-accuracy positioning system, it is possible to coherently combine the measurements to generate high resolution radar images using Synthetic Aperture Radar (SAR) algorithms.

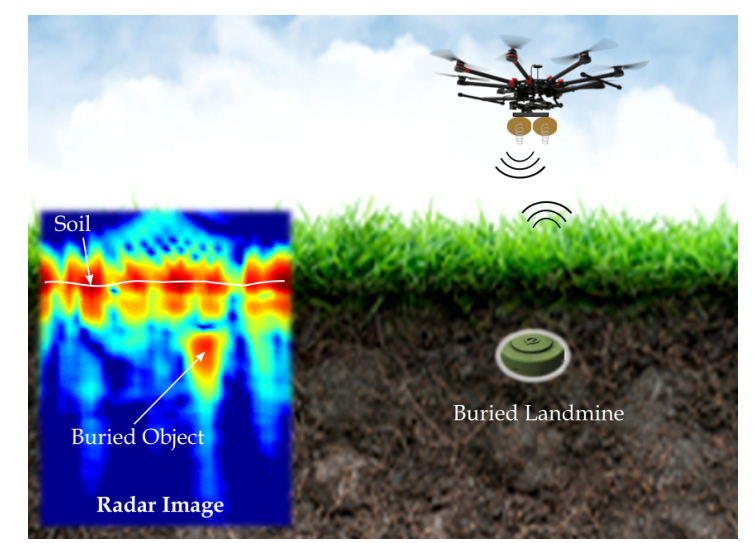

Fig. 1. Scheme of the proposed approach for landmine detection. 
Although some systems consisting of a radar onboard a UAV have been already developed, they are not able neither to detect buried targets (only targets on the surface) [7] nor to create underground images. To the best of the authors' knowledge, this is the first UAV-mounted GPR system that has been experimentally validated and is able not only to detect buried targets, but also to create high-resolution underground images (thanks to its accurate positioning system).

\section{System ARChitecture}

The proposed system is mainly composed by a GPR mounted on a UAV. The ultimate goal is that the UAV autonomously follows a predefined flight path over the area to be inspected. During the flight, geo-referred radar measurements are sent to a ground control station in real time, where they are processed to generate images of the underground. The first prototype of the proposed system includes the following subsystems.

- Flight control subsystem: UAV flight controller and common positioning sensors (inertial measurement unit, barometer, and Global Navigation Satellite System -GNSS- receiver).

- Communication subsystem: radio transmitter and receiver modules working at $433 \mathrm{MHz}$ and wireless network at $2.4 \mathrm{GHz}$, in order to avoid interference with the GPR working frequencies.

- Accurate positioning subsystem: Real Time Kinematic (RTK) system and a LIDAR (LIght Detection And Ranging) rangefinder, providing $\mathrm{cm}$-level accuracy positioning information. The RTK system is composed by two RTK beacons: one on the UAV and another at a fixed position on the ground. The latter sends GNSS corrections to the former. The LIDAR rangefinder, which is mounted pointing downwards, is used to improve the accuracy in height.

- Radar subsystem: radar module and antennas. The radar module transmits a short gaussian pulse at C-band (from 3.1 to $5.1 \mathrm{GHz}$ ), providing a high range resolution at the expense of a worst penetration depth (since attenuation increases with frequency). The antennas are two circularly polarized helix antennas with reverse handedness, well-matched at the radar frequency band and with a beamwidth of around $47^{\circ}$.

- Ground control station, which is a laptop, where the geo-referred radar measurements are received and represented in real time. Since geo-referring accuracy is better than a quarter wavelength, a SAR algorithm could be used to obtain high resolution radar images.

An scheme of these subsystems and the connections between them is shown in Fig. 2. All these subsystems on board the UAV are shown in Fig. 3.

Regarding the methodology, in this initial validation, the envelope of the geo-referred radar measurements is used to generate the radar images. Since the radar is an Ultra-Wide-Band (UWB) impulse radar that transmits gaussian pulses, the envelope is calculated using the Hilbert transform

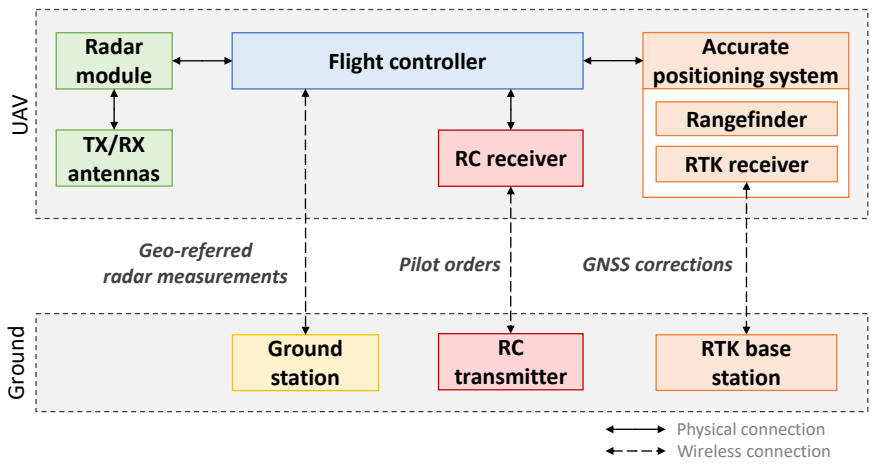

Fig. 2. Scheme of the main subsystems that compose the prototype.

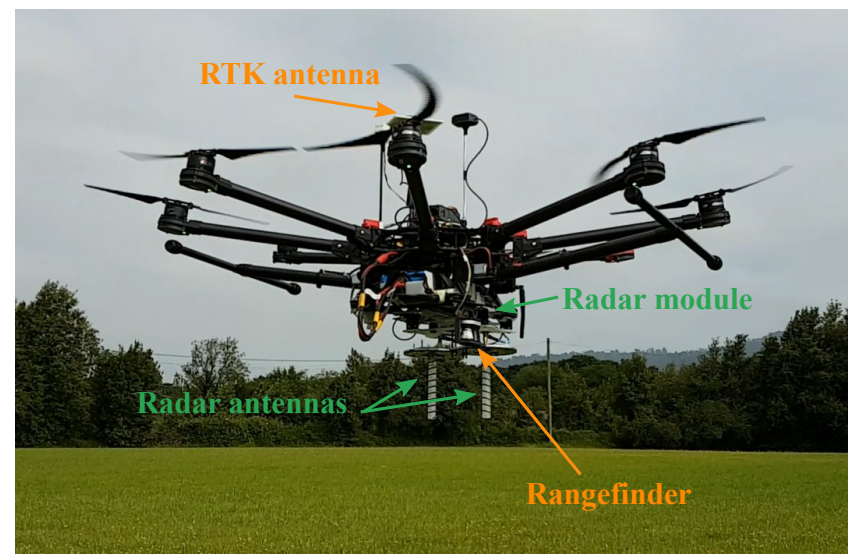

Fig. 3. UAV-mounted GPR prototype.

of the measured signal. For each measurement $u(\boldsymbol{r}, t)$, the Hilbert transform $\hat{u}(\boldsymbol{r}, t)=\mathcal{H}[u(\boldsymbol{r}, t)]$ is calculated to obtain the analytic signal $u_{a}(\boldsymbol{r}, t)$. The envelope, $u_{\text {env }}(\boldsymbol{r}, t)$, is the magnitude of the analytic signal, as shown in (1). For each measurement taken along the UAV flight path, the envelope is plotted to create a two-dimensional radar image.

$$
u_{e n v}(\boldsymbol{r}, t)=\left|u_{a}(\boldsymbol{r}, t)\right|=|u(\boldsymbol{r}, t)+j \hat{u}(\boldsymbol{r}, t)|
$$

\section{EXPERIMENTAL VALIDATION}

Experimental validation has been performed in several stages. Since digging is not allowed in the airfield, a plastic box filled with sand has been used (burying the objects inside it). Therefore, first tests were devoted to analyze the performance of the radar for detecting objects inside a sandbox: initially, in a controlled scenario (a planar measurement range) [8], and then, with a manual positioning scanner. Once the radar was tested, first flight tests were conducted.

\section{A. Manual Scanning}

For the initial tests, several objects were buried in a box of size $(45,62,32) \mathrm{cm}$, filled with a layer of $21 \mathrm{~cm}$ of sand (with relative permittivity $\varepsilon_{r} \approx 3.5$ ). Once the radar was tested in a controlled environment [8], it was tested using the manual scanner shown in Fig. 4 in order to analyze whether positioning errors affect notably the radar images. Measurements were taken along a 1-m long aperture, sampled every $2 \mathrm{~cm}$. 
The envelope of the radar measurements is shown in Fig. 5 when a metallic disk of $9-\mathrm{cm}$ radius and $1-\mathrm{cm}$ thickness is buried at $8 \mathrm{~cm}$ depth (Fig. 5a), and when a plastic disk of $9-\mathrm{cm}$ radius and $3-\mathrm{cm}$ thickness is buried at $7 \mathrm{~cm}$ depth (Fig. 5b). It can be noticed that both objects are detected deeper than their true locations since the permittivity of the sand is not taken into account to represent the results [9]. The sand-soil interface also appears downshifted. Furthermore, due to the low dielectric contrast between the plastic object and the sand, this object is barely distinguishable in the radar image.

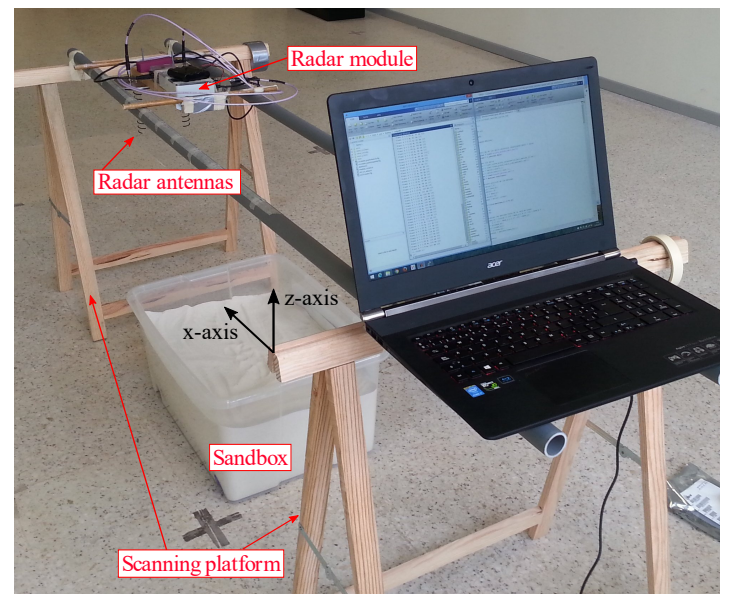

Fig. 4. Setup for the manual scanning tests.

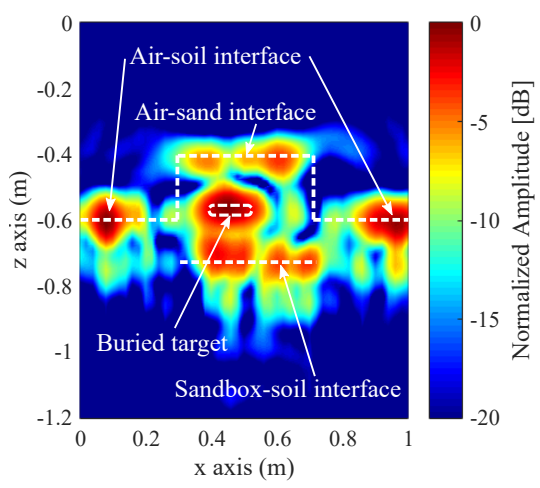

(a)

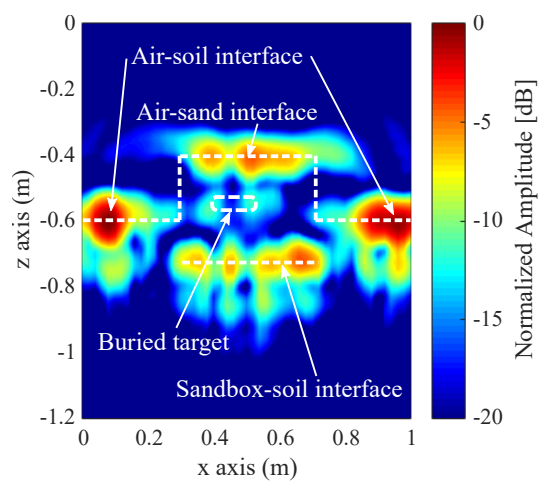

(b)

Fig. 5. Envelope of radar measurements when an object is buried in the sandbox: metallic (a) and plastic (b) object.

\section{B. Initial Flight Tests}

For the flight tests, a bigger sandbox of $(56,78,43) \mathrm{cm}$ size was used (in order to be able to bury the targets deeper if needed). The box was placed on the grass of the airfield and covered with a canvas as shown in Fig. 6.

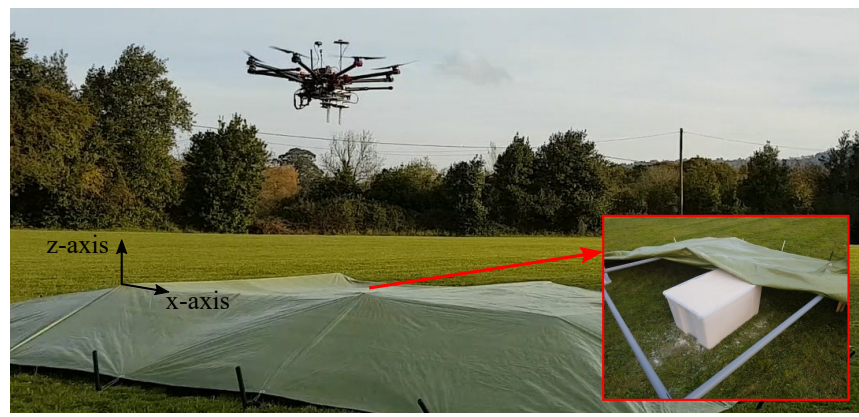

Fig. 6. Setup for the flight tests.

First, the metallic disk used in the previous subsection was buried at $12 \mathrm{~cm}$ depth. In the radar envelope, shown in Fig. $7 \mathrm{a}$, the air-sand interface, the object and the air-soil interface are clearly distinguishable. The buried target and the sand-soil interface appear also downshifted as the sand permittivity ( $\varepsilon_{r} \approx 2.5$ in this case) is not considered. Furthermore, the two plastic bars that support the canvas are detected at around $60 \mathrm{~cm}$ and $70 \mathrm{~cm}$ away from the left and right sides of the box.

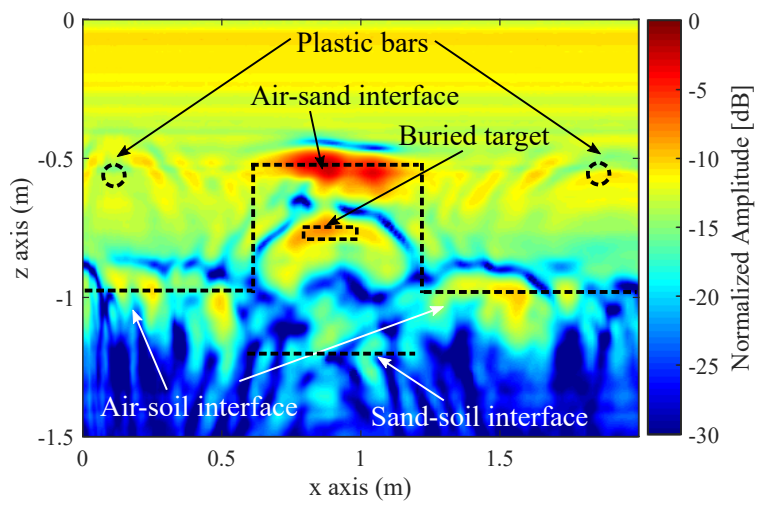

(a)

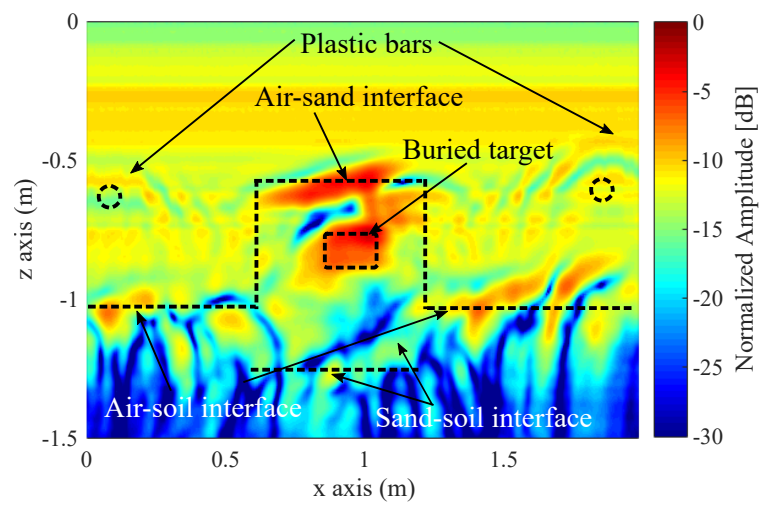

(b)

Fig. 7. Envelope of radar measurements taken with the prototype when an object is buried in the sandbox: metallic (a) and plastic (b) object. 
Next, a plastic cylinder (with $9-\mathrm{cm}$ radius and $9.5-\mathrm{cm}$ thickness, filled with foam) was buried at $10 \mathrm{~cm}$ depth. Due to the large size of this target and the dielectric contrast between the foam and the sand, it is even better detected in the radar envelope (shown in Fig. 7b). In this case, the sand-soil interface under the sandbox is also slightly better distinguishable.

In the second measurement the UAV flight path was not as parallel to the ground as in the first one. Nevertheless, the information provided by the LIDAR altimeter could be used to correct this deviation in height if needed.

\section{CONCLUSION}

In this contribution, a novel system to detect subsurface targets has been presented. It is mainly composed by a GPR on board a UAV. Its ultimate goal is to autonomously inspect a predefined area, sending the geo-referred radar measurements to a ground station to be processed. The proposed system overcomes some limitations of current state-of-the-art systems regarding speed, security and cost. Furthermore, as it has been shown in this contribution, it is able to detect both metallic and dielectric targets, which is useful for detecting landmines or IEDs with low metallic content. New improvements in the system, such as the use of SAR algorithms to generate high resolution images from the radar measurements, are currently in progress and will be presented at the conference.

\section{ACKNOWLEDGMENT}

This work has been partially supported by Government of Spain under projects TEC2014-55290-JIN (PORTEMVISION) and TEC2015-73908-JIN, and grants RYC-2016-20280 and FPU15/06341; by the Gobierno del Principado de Asturias through IDI/2017/000095; and by the Galician Regional Government under project GRC2015/018 and under agreement for funding AtlantTIC.

\section{REFERENCES}

[1] L. Robledo, M. Carrasco, and D. Mery, "A survey of landmine detection technology," International Journal of Remote Sensing, vol. 30, pp. 2399-2410, 2009.

[2] H. M. Jol (Editor), Ground Penetrating Radar: Theory and Applications, 1st. Ed. Amsterdam: Elsevier, 2009.

[3] G. Liu, Y. Wang, J. Li, and M. R. Bradley, "SAR imaging for a forward-looking GPR system," in AeroSense 2003, pp. 322-333, 2003.

[4] E. M. Rosen, and E. Ayers, "Assessment of down-looking GPR sensors for landmine detection," in Proc. SPIE 5794, Detection and Remediation Technologies for Mines and Minelike Targets X, pp. 423-434, 2005.

[5] B. Gonzalez, et al., "Airborne systems and detection methods for the localization and production of images of buried objects and characterization of the subsurface composition," ES Patent 2577403, 2016.

[6] Minekafon project, http://minekafon.org/. Last access: 01/02/2018.

[7] C. J. Li, and H. Ling, "High-resolution downward-looking radar imaging using a small consumer drone," in 2016 IEEE AP-S Symposium on Antennas and Propagation, pp. 2037-2038, 2016.

[8] M. Garcia-Fernandez, et al., "Experimental Validation of a GPR Imaging System,", in 2017 11th European Conference on Antennas and Propagation (EUCAP), pp. 3580-3584, 2017.

[9] Y. Alvarez, et al., "SAR-based technique for soil permittivity estimation, International Journal of Remote Sensing, vol. 38, no. 18, pp. 5168-5185, 2017. 